\title{
Retinamorphic color Schrödinger metamedia
}

\author{
V. Labunets ${ }^{1}$, I. Artemov ${ }^{1}$, V. Chasovskikh ${ }^{1}$, E. Ostheimer ${ }^{2}$ \\ ${ }^{1}$ Ural State Forest Engineering University, 620100, Ekaterinburg, Russia \\ ${ }^{2}$ Capricat LLC, Pompano Beach, Florida, USA
}

\begin{abstract}
In this work, we use quantum color cellular automata to study pattern formation and image processing in quantum-diffusion Schrödinger systems with triplet-valued (color-valued) diffusion coefficients. Triplet numbers have the real part and two imaginary parts (with two imaginary units $\varepsilon^{1}$ and $\varepsilon^{2}$, where $\varepsilon^{3}=1$ ). They form 3-D triplet algebra. Discretization of the Schrödinger equation gives quantum color cellular automata with various triplet-valued physical parameters. The process of excitation in these media is described by the color Schrödinger equations with the wave functions that have values in triplet algebras. The color Schrödinger metamedia can be used for creation of the eye-prosthesis. The color metamedium suggested can serve as the prosthesis prototype for perception of the color images.
\end{abstract}

Keywords: Color Schrödinger equation; color Schrödinger transform; color metamedia; cellular automata; silicon eye; color image processing

\section{Introduction}

Diffusion and Schrödinger equations (linear and nonlinear) [1,2] and real-valued Gauss, complex-valued Fresnel and Schrödinger transforms associated with them are important members of the family of methods for image processing, computer vision, and computer graphics. Schrödinger transform of image as a new tool for image analysis was first given in [2]. Neural networks and cellular automata (in form of a media) which are compatible with the theory of quantum mechanics and demonstrate the particle-wave nature of information have been analyzed in [3-5]. The studying of processes in such metamedia is very important for many branches of the system theory. There is no general theory of the metamedia yet, and every particular example of similar media, usually provides us with the examples of new dynamic or self-organization types.

In this work, we apply quantum cellular automata to study pattern formation and image processing in quantum-diffusion Schrödinger metamedia with triplet-valued diffusion coefficients. Triplet (color) numbers [6] contain one real and two imaginary components with two hyper-imaginary units $\varepsilon$ and $\varepsilon^{2}$ and the following property $\varepsilon^{3}=1: \mathrm{C}=r+g \varepsilon^{1}+b \varepsilon^{2}$, where $r, g, b$ are real numbers. The numbers $\mathrm{C}=r+g \varepsilon^{1}+b \varepsilon^{2}$ are called triplet or color numbers. They form a 3-D triplet (color) algebra $\mathrm{A}_{3}(\varepsilon)=\mathrm{A}_{3}\left(\mathbf{R} \mid 1, \varepsilon^{1}, \varepsilon^{2}\right):=\left\{\mathrm{C}=r+g \varepsilon^{1}+b \varepsilon^{2} \mid r, g, b \in \mathbf{R}\right\}$. If the diffusion coefficient in the Fourier diffusion or Planck's constant in the Schrödinger equations are a triplet number $\mathrm{D}=r+g \varepsilon^{1}+b \varepsilon^{2}$ then both equations are turned into the color Schrodinger equation. It describes the process of excitement in the so-called color Schrodinger metamedium with $\mathrm{A}_{3}(\varepsilon)$-valued (color) wave function $\varphi(x, y, t)=\varphi_{r}(x, y, t)+\varphi_{g}(x, y, t) \varepsilon^{1}+\varphi_{b}(x, y, t) \varepsilon^{2} \mid$.

In this work, we study properties of the color Schrödinger excitable metamedium in the form of a cellular automaton. The more detailed information about cellular automata can be found in [7]. The automaton's cells are located inside a 2D array. They can perform basic operations with triple (color) numbers (in color algebra $\mathrm{A}_{3}\left(\mathbf{R} \mid 1, \varepsilon^{1}, \varepsilon^{2}\right)$ ). These cells are able to inform the neighboring cells about their states. Such media possess large opportunities in processing of color images in comparison with the ordinary diffusion media with the real-valued diffusion coefficient.

The rest of the paper is organized as follows: in Section 2, the object of the study (the color Schrödinger equation) is described. In Section 3, a brief introduction to mathematical background (color algebra $\mathrm{A}_{3}=\mathrm{A}_{3}\left(\mathbf{R} \mid 1, \varepsilon^{1}, \varepsilon^{2}\right)$ of triplet numbers $\mathrm{C}=r+g \varepsilon^{1}+b \varepsilon^{2}$ ) is given (subsection 3.1) in order to understand the concept behind the proposed method. In subsection 3.2, the proposed method based on color Schrödinger equations is explained. Next, we defined Schrödinger transform of color image, discussed its properties. In Section 4, the basic color metamedia (the color Schrödinger-Euclidean, color SchrödingerMinkowskian, color Schrödinger-Galilean and color Schrödinger-Yaglom) are devised and analyzed in detail. The simulation result and algorithm complexity are demonstrated too. Finally, we gave our conclusion in Section 5.

\section{The object of the study}

In this work, we apply quantum cellular automata to study pattern formation and image processing in color quantumdiffusion Schrödinger metamedia with triplet-valued diffusion coefficients:

$$
\frac{\partial \varphi(x, y, t)}{\partial t}=\mathrm{D}\left(\frac{\partial^{2} \varphi(x, y, t)}{\partial x^{2}}+\frac{\partial^{2} \varphi(x, y, t)}{\partial y^{2}}\right)+f(x, y, t),
$$

where $\varphi(x, y, t)=\varphi_{r}(x, y, t)+\varphi_{g}(x, y, t) \varepsilon^{1}+\varphi_{b}(x, y, t) \varepsilon^{2}$ is a color wave function that describes excitement of medium, $f(x, y, t)=f_{r}(x, y, t)+f_{g}(x, y, t) \varepsilon^{1}+f_{b}(x, y, t) \varepsilon^{2}$ is an exciting color source (input color signal) and $\mathrm{D}=r+g \varepsilon^{1}+b \varepsilon^{2}$ is colorvalued diffusion coefficient. It describes the process of excitement in the so-called color Schrodinger metamedium with $\mathrm{A}_{3}(\varepsilon)$ valued (color) wave function $\varphi(x, y, t)$. Discretization of the color Schrödinger equation gives a color quantum Schrödinger 
cellular automaton with various triple-valued physical parameters. Their microelectronic realizations appear to be a programmable Schrodinger metamedia [8]. The main purpose of this work is the investigation of time evolution for color Schrödinger metamedia in the form of quantum cellular automata with triplet diffusion coefficients. The automaton's cells are located inside a $2 \mathrm{D}$ array. They can perform basic operations with triple (color) numbers (in color algebra $\mathrm{A}_{3}\left(\mathbf{R} \mid 1, \varepsilon^{1}, \varepsilon^{2}\right)$ ). These cells are able to inform the neighboring cells about their states. Such media possess large opportunities in processing of color images in comparison with the ordinary diffusion media with the real-valued diffusion coefficients. The latter media are used for creation of the eye-prosthesis (so called the "silicon eye"). The medium suggested can serve as the prosthesis prototype for perception of the color images [9-15].

\section{Methods}

\subsection{Mathematical background. Triplet algebra}

Let us consider the algebraic and geometric properties of the triplet algebra $\mathrm{A}_{3}=\mathrm{A}_{3}\left(\mathbf{R} \mid 1, \varepsilon^{1}, \varepsilon^{2}\right):=$ $=\left\{\mathrm{C}=r+g \varepsilon^{1}+b \varepsilon^{2} \mid r, g, b \in \mathbf{R}\right\}$. The addition and product of two triplet numbers $\mathrm{C}_{1}=\left(r_{1}+g_{1} \varepsilon+b_{1} \varepsilon^{2}\right)$ and $\mathrm{C}_{2}=\left(r_{2}+g_{2} \varepsilon+b_{2} \varepsilon^{2}\right)$ are given by $[6]$ :

$$
\begin{aligned}
& \mathrm{C}_{1}+\mathrm{C}_{2}=\left(r_{1}+g_{1} \varepsilon^{1}+b_{1} \varepsilon^{2}\right)+\left(r_{2}+g_{2} \varepsilon^{1}+b_{2} \varepsilon^{2}\right)=\left(r_{1}+r_{2}\right)+\left(g_{1}+g_{2}\right) \varepsilon^{1}+\left(b_{1}+b_{2}\right) \varepsilon^{2}, \\
& \mathrm{C}_{1} \cdot \mathrm{C}_{2}=\left(r_{1}+g_{1} \cdot \varepsilon+b_{1} \cdot \varepsilon^{2}\right) \cdot\left(r_{2}+g_{2} \cdot \varepsilon+b_{2} \cdot \varepsilon^{2}\right)=\left(r_{1} r_{2}+b_{1} g_{2}+g_{1} b_{2}\right)+\left(g_{1} r_{2}+r_{1} g_{2}+b_{1} b_{2}\right) \varepsilon+\left(b_{1} r_{2}+g_{1} g_{2}+r_{1} b_{2}\right) \varepsilon^{2} .
\end{aligned}
$$

It is useful to introduce the following triplet numbers $\mathbf{e}_{l u m}:=\left(1+\varepsilon+\varepsilon^{2}\right) / 3, \quad \mathbf{E}_{c h r}:=\left(1+\omega_{3} \varepsilon^{2}+\omega_{3}^{2} \varepsilon\right) / 3, \quad$ where $\omega_{3}=\exp \left(i_{-} \cdot 2 \pi / 3\right)$. It is easy to check $\mathbf{e}_{l u m}^{2}=\mathbf{e}_{l u m}, \mathbf{E}_{c h r}^{2}=\mathbf{E}_{c h r}, \mathbf{e}_{l u m} \mathbf{E}_{c h r}=\mathbf{E}_{c h r} \mathbf{e}_{l u m}=0$. Hence, $\mathbf{e}_{l u m}, \mathbf{E}_{c h r}$ are orthogonal idempotents (projectors) and every triplet (color) number $\mathrm{C}=r+g \varepsilon+b \varepsilon^{2}$ can be represented in the form of the linear combination of a "scalar" $a_{l u m} \cdot \mathbf{e}_{l u m}$ and "complex" $z_{c h r} \cdot \mathbf{E}_{c h r}$ components $\mathrm{C}=a_{l u m} \cdot \mathbf{e}_{l u m}+z_{c h r} \cdot \mathbf{E}_{c h r}=\left(a_{l u m}, z_{c h r}\right)$ in the idempotent basis $\left\{\mathbf{e}_{l u m}, \mathbf{E}_{c h r}\right\}$, where $a_{l u m} \cdot \mathbf{e}_{l u m} \equiv \mathrm{C} \cdot \mathbf{e}_{l u m}, z_{c h r} \cdot \mathbf{E}_{c h r}=\mathrm{C} \cdot \mathbf{E}_{c h r}$, because

$$
\begin{aligned}
& \mathrm{C} \cdot \mathrm{e}_{l u}=\left(a_{l u} \cdot \mathbf{e}_{l u}+z_{c h} \cdot \mathbf{E}_{c h}\right) \cdot \mathbf{e}_{l u}=a_{l u} \cdot \mathbf{e}_{l u}^{2}+z_{c h} \cdot \mathbf{E}_{c h} \mathbf{e}_{l u}=a_{l u} \cdot \mathbf{e}_{l u} \overline{\bar{F}} \\
& \mathrm{C} \cdot \mathbf{E}_{c h}=\left(a_{l u} \cdot \mathbf{e}_{l u}+z_{c h} \cdot \mathbf{E}_{c h}\right) \cdot \mathbf{E}_{c h}=a_{l u} \cdot \mathbf{e}_{l u} \mathbf{E}_{c h}+\mathbf{Z}_{c h} \cdot \mathbf{E}_{c h}^{2}=z_{c h} \cdot \mathbf{E}_{c h} .
\end{aligned}
$$

We will call real numbers $a_{\text {lum }} \in \mathbf{R}$ the luminance numbers and complex numbers $z_{c h r} \in \mathbf{C}$ - the chromatic numbers. Obviously,

$$
\begin{aligned}
& a_{l u m} \cdot \mathbf{e}_{l u m}=\mathrm{C} \cdot \mathbf{e}_{l u m}=\left(r+g \varepsilon^{1}+b \varepsilon^{2}\right) \frac{1+\varepsilon^{1}+\varepsilon^{2}}{3}=(r+g+b) \frac{1+\varepsilon^{1}+\varepsilon^{2}}{3}, \\
& z_{c h r} \cdot \mathbf{E}_{c h r}=\mathrm{C} \cdot \mathbf{E}_{c h r}=\left(r+g \varepsilon^{1}+b \varepsilon^{2}\right) \frac{1+\omega^{1} \varepsilon^{1}+\omega^{2} \varepsilon^{2}}{3}=\left(r+g \omega^{1}+b \omega^{2}\right) \frac{1+\omega^{1} \varepsilon^{1}+\omega^{2} \varepsilon^{2}}{3} .
\end{aligned}
$$

Hence, $a_{l u m}=r+g+b, z_{c h r}=r+g \omega^{1}+b \omega^{2}=\left(r-\frac{g+b}{2}\right)+i_{-} \frac{\sqrt{3}}{2}(g-b)$. In the new duplex representation two main arithmetic operations have the simplest form:

$$
\begin{aligned}
& \mathrm{C}+\mathrm{B}=\left(a_{l u m} \cdot \mathbf{e}_{l u m}+z_{c h r} \cdot \mathbf{E}_{c h r}\right)+\left(b_{l u m} \cdot \mathbf{e}_{l u m}+w_{c h r} \cdot \mathbf{E}_{c h r}\right)=\left(a_{l u}+b_{l u}\right) \cdot \mathbf{e}_{l u m}+\left(z_{c h r}+w_{c h r}\right) \cdot \mathbf{E}_{c h r}, \\
& \mathrm{C} \cdot \mathrm{B}=\left(a_{l u m} \cdot \mathbf{e}_{l u m}+z_{c h r} \cdot \mathbf{E}_{c h r}\right) \cdot\left(b_{l u m} \cdot \mathbf{e}_{l u m}+w_{c h r} \cdot \mathbf{E}_{c h r}\right)=\left(a_{l u} b_{l u}\right) \cdot \mathbf{e}_{l u m}+\left(z_{c h r} w_{c h r}\right) \cdot \mathbf{E}_{c h r} .
\end{aligned}
$$

Consequently, a color algebra $\mathrm{A}_{3}(\varepsilon)$ is the direct sum of real $\mathbf{R}$ and complex $\mathbf{C}$ fields: $\mathrm{A}_{3}(\varepsilon)=\mathbf{R} \cdot \mathbf{e}_{l u}+\mathbf{C} \cdot \mathbf{E} c h=\mathbf{R} \oplus \mathbf{C}$. It is known that every 2-D complex number $\mathbf{z}=x+i y$ can be represented geometrically by the modulus $\mathbf{z}=x+i y$ $\rho=|\mathbf{z}|=\sqrt{x^{2}+y^{2}}$ and by the polar angle $\theta=\operatorname{arctg}(x / y)$. The modulus $\rho$ is multiplicative and the polar angle $\theta$ is additive upon the multiplication of ordinary complex numbers. The triplet numbers introduced in this section have the form $\mathcal{C}=r+g \varepsilon^{1}+b \varepsilon^{2}$, the variables $r, g$ and $b$ being real numbers. In a geometric representation, the triplet number $\mathcal{C}=r+g \varepsilon^{1}+b \varepsilon^{2}$, is represented by the point $C(r, g, b)$, or as a 3 -D vector with coordinates $(r, g, b)$ in the 3 -D color space $\mathbf{R}_{\text {col }}^{3}$ (see Fig. 1).

Let the point $C$ be the point of the origin of the $R, G, B$ axes, and $T_{A c h}$ will be the line, which contains the points with equal coordinates $r=g=b$ (it is called an achromatic diagonal). The luminance numbers $a_{\text {lum }}$ lie on this achromatic diagonal. Also let $\Delta_{M}\left(a_{l u}\right)$ be the plane $r+g+b=a_{l u m}$ that is perpendicular to an achromatic axis $T_{A c h}$; this plane intersects it on a range $a_{\text {lum }}$ from the point of origin $C$. It is called a chromatic plane. It contains chromatic numbers $Z_{c h r}$. 


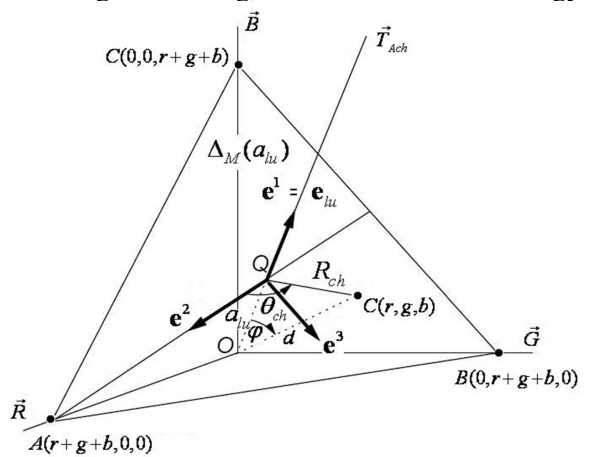

a)

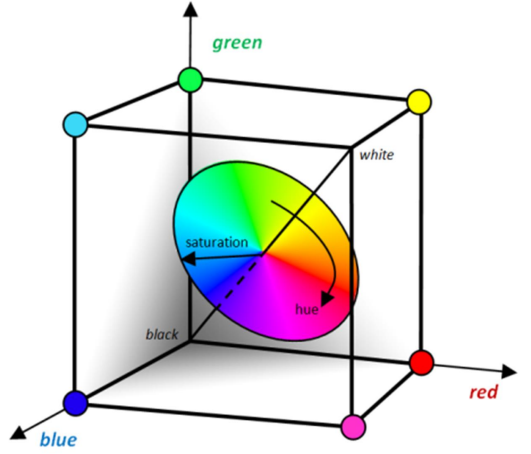

b)

Fig. 1. a) The geometrical representation of a triplet number $\mathrm{C} \equiv r+g \varepsilon+b \varepsilon^{2}$ in the form of a $3 \mathrm{D}$ vector $\mathrm{C} \equiv(r, g, b) \in \mathbf{R}_{c o l}^{3}$ or as a point $\mathrm{C} \equiv C(r, g, b) \in \mathbf{R}_{c o l}^{3}$ in 3-D color space $\mathbf{R}_{c o l}^{3}$. The geometrical characteristics have the following values: $a_{l u m}=(r+g+b) / \sqrt{3}$, $d=\sqrt{r^{2}+g^{2}+b^{2}}, R_{c h r}=\sqrt{d^{2}-a_{l u m}^{2}}, \theta_{c h r}=\arg \left(z_{c h r}\right)$ b) the color cube, it's achromatic diagonal and chromatic plane.

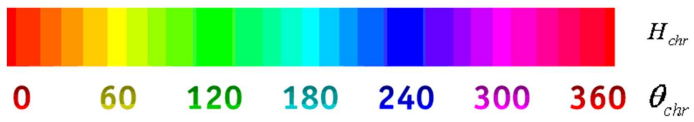

Fig. 2. The relation between an angle $\theta_{c h r}(x, y, t)$ and hue $H_{c h r}(x, y, t)$.

Obviously, a vector $\mathrm{C} \equiv r+g \varepsilon+b \varepsilon^{2}=(r, g, b)$ can be described: 1$)$ by the projection $a_{\text {lum }}$ of a line segment $O C$ on a line $T_{A c h}$, i.e. by the luminance component and 2) by a complex number $z_{c h r}$ in the chromatic plane. Besides, the absolute value of this number appears to be the range $\left|z_{c h r}\right|$ from $C(r, g, b)$ to this line, i.e. it describes the saturation (which is marked by the symbol $S_{c h r}=\left|z_{c h r}\right|$ ) of a triplet number $\mathrm{C}=r+g \varepsilon+b \varepsilon^{2}$ and the azimuth angle $\theta_{c h r}=\arg \left(z_{c h r}\right)$ represents its color hue (which we can mark by the symbol $\left.H_{c h r}=\theta_{c h r}=\arg \left(z_{c h r}\right)\right)$ according to Fig. 2.

\subsection{The generalized Schrödinger equation and cellular automata}

Let a diffusion coefficient $\mathrm{D}=r+g \varepsilon^{1}+b \varepsilon^{2}$ in the Schrödinger equation

$$
\frac{\partial \varphi(x, y, t)}{\partial t}=\left(r+g \varepsilon^{1}+b \varepsilon^{2}\right) \cdot\left(\frac{\partial^{2} \varphi(x, y, t)}{\partial x^{2}}+\frac{\partial^{2} \varphi(x, y, t)}{\partial y^{2}}\right)+f(x, y, t),
$$

be a triplet number, where $\varphi(x, y, t)=\varphi_{r}(x, y, t)+\varphi_{g}(x, y, t) \varepsilon^{1}+\varphi_{b}(x, y, t) \varepsilon^{2}$ is a color wave function that describes excitement of medium, $f(x, y, t)=f_{r}(x, y, t)+f_{g}(x, y, t) \varepsilon^{1}+f_{b}(x, y, t) \varepsilon^{2}$ is an exciting color source (input color signal). Color wave function $\varphi(x, y, t)$ describes time evolution of state $\varphi(x, y, t)$ (in terms of triplet numbers) of a metamedium point with coordinate ( $x, y$ ). If $\mathrm{D} \equiv D_{c l}=r \in \mathbf{R}$ is a real number and $\varphi(x, y, t)=\varphi_{r}(x, y, t)$ then (2) is an ordinary diffusion (or heat) equation for the real ordinary medium (we will call one as the Fourier-Gauss medium). If $\mathrm{D} \equiv i D_{q u} \in \mathbf{C}$ is an imaginary number and $\varphi(x, y, t)=\varphi_{c l}(x, y, t)+i \varphi_{q u}(x, y, t)$ then (2) becomes an ordinary Schrödinger equation with the Plank's constant $i D_{q u} \in i / 2 m$ for ordinary quantum Schrödinger medium. If $\mathrm{D}=D_{c l}+i D_{q u} \in \mathrm{A}_{2}(\mathbf{R} \mid i)$ and $\varphi(x, y, t)=\varphi_{c l}(x, y, t)+i \varphi_{q u}(x, y, t)$ then $(2)$ is bichromatic Schrödinger equation for bichromatic quantum Schrödinger metamedium [16]. It is a generalization of both diffusion and Schrödinger metamedia.

In case of zero initial conditions, we can write the solution of (2) in the form of the Cauchy integral:

$$
\varphi(x, y, t)=\int_{0}^{t} \frac{1}{(2 \sqrt{\pi \mathrm{D}(t-\tau)})^{2}}\left(\int_{-\infty}^{+\infty} \int_{-\infty}^{+\infty} e^{-\frac{(x-\xi)^{2}+(y-\eta)^{2}}{4 \mathrm{D}(t-\tau)}} f(\xi, \eta, \tau) d \xi d \eta\right) d \tau .
$$

This integral we will call the color Schrödinger transform (GST) of the initial image $f(x, y, t)$. If $i D_{q u} \in i / 2 m \in \mathbf{C}=\mathrm{A}_{2}\left(\mathbf{R} \mid i_{-}\right)$, then GST is ordinary Schrödinger transform [1-5].

Let us introduce a 2-D regular lattice with nodes $\left(x_{n}, y_{m}, t_{k}\right)$, where $x_{n+1}=x_{n}+h, y_{m+1}=y_{m}+h$ and $t_{k+1}=t_{k}+\tau$. Here $h$ and $\tau$ are spaces between nodes on the space $\mathbf{Z}_{S p}^{2} \subset \mathbf{R}^{2}$ and time $\mathbf{Z}_{t} \subset \mathbf{R}_{t}$ lattices, respectively. For discrete Laplacian we use the following approximation:

$$
\begin{aligned}
& d^{2} \varphi / d x^{2}=\varphi\left(x_{n}+1, y_{m}, t_{k}\right)+\varphi\left(x_{n}-1, y_{m}, t_{k}\right)-2 \varphi\left(x_{n}, y_{m}, t_{k}\right), \\
& d^{2} \varphi / d y^{2}=\varphi\left(x_{n}, y_{m}+1, t_{k}\right)+\varphi\left(x_{n}, y_{m}-1, t_{k}\right)-2 \varphi\left(x_{n}, y_{m}, t_{k}\right), \\
& d^{2} \varphi / d t=\varphi\left(x_{n}, y_{m}, t_{k}+1\right)-\varphi\left(x_{n}, y_{m}, t_{k}\right) .
\end{aligned}
$$


As a result, we get the 2-D discrete color Schrödinger equation

$$
\begin{aligned}
& \varphi\left(x_{n}, y_{m}, t_{k}+1\right)=\varphi\left(x_{n}, y_{m}, t_{k}\right)+ \\
& +\mathrm{D} \cdot\left[\varphi\left(x_{n}+1, y_{m}, t_{k}\right)+\varphi\left(x_{n}-1, y_{m}, t_{k}\right)+\varphi\left(x_{n}, y_{m}+1, t_{k}\right)+\varphi\left(x_{n}, y_{m}-1, t_{k}\right)-4 \varphi\left(x_{n}, y_{m}, t_{k}\right)\right] .
\end{aligned}
$$

Now, we give the definition of a 2-D "cellular space" (2-D regular lattice) in which the cellular automaton is defined. A regular lattice $\mathbf{Z}_{S p}^{2} \subset \mathbf{R}_{S p}^{2}$ consists of a set of cells (elementary automata, or electrical circuits Aut ), which homogeneously cover a 2-D Euclidean space. Each cell is labeled by its position $\mathrm{A} \boldsymbol{w} t\left(x_{n}, y_{m}\right)=\mathrm{A} \boldsymbol{w} t(n, m),(n, m) \in \mathbf{Z}_{S p}^{2}$

Regular, discrete, infinite network consisting of a large number of simple identical elements in the form of elementary automata $\mathrm{A} \boldsymbol{u} t(n, m)$ a copy of which will take place at each node $(n, m)$ of the net is called the cellular automaton (see Fig.2 and Fig.3a in [16] ). Each so decorated note will be called a cell Aut $(n, m)$ and will communicate with a finite number of other cells Aut $(i, k)$, which determine its neighborhood $(i, k) \in \mathbf{M}(m, n)$, geometrically uniform $\mathbf{M}(m, n) \equiv \mathbf{M}, \forall \mathbf{M}(m, n) \in \mathbf{Z}_{s p}^{2} . \quad$ The neighborhood of the cell Avt $(n, m)$ (including the cell itself or not, in accordance with convention) is the set of all the cells A $\boldsymbol{u} t(i, k),(i, k) \in \mathbf{M}(m, n)$ of the network which will locally determine the evolution of A $\boldsymbol{u} t(n, m)$. This local communication, which is deterministic, uniform and synchronous determines a global evolution of the cellular automaton, along discrete time steps $t_{k+1}=t_{k}+\tau$.

In the case of $\mathbf{Z}_{S p}^{2}$, the classical neighborhoods are the von Neumann and Moore ones. They are known as the nearest neighbors neighborhoods, and defined according to the usual norms and the associated distances. More precisely, for $(i, j) \in \mathbf{Z}_{s p}^{2}$ , $\|(i, j)\|_{1}=|i|+|j|$ and $\|(i, j)\|_{\infty}=\max (|i|,|j|)$ will denote ${ }_{1}$ - and ${ }_{\infty}$-norm respectively. Let $\rho_{1}$ and $\rho_{\infty}$ be the associated distances. Then Von Neumann and Moore neighborhoods (Fig.2) are $\mathbf{M}_{+}(m, n):=\left\{(i, k) \mid \rho_{1}((m, n),(i, k)) \leq 1\right\}$ and $\mathbf{M}(m, n):=\left\{(i, k) \mid \rho_{\infty}((m, n),(i, k)) \leq 1\right\}$, respectively. To each cell Aut $(n, m)$ we assign an $\mathrm{A}_{2}(\mathbf{R} \mid i)$-valued state $\varphi(n, m, k)=\varphi\left(x_{n}, y_{m}, t_{k}\right)$ (i.e., the media's excitement). The dynamics of the cellular automaton are determined by a local transition rule, which specifies the new state $\varphi(n, m, k+1)=\varphi\left(x_{n}, y_{m}, t_{k+1}\right)$ of a cell as a function of its interaction Von Neumann neighborhood configuration, according to (5), i.e.,

$$
\varphi(n, m, k+1)=\varphi(n, m, k)+\mathrm{D} \cdot[\varphi(n+1, m, k)+\varphi(n-1, m, k)+\varphi(n, m+1, k)+\varphi(n, m-1, k)-4 \varphi(n, m, k)] .
$$

This rule shows us the relation between a state $\varphi(n, n, k+1)$ of the cell A $\boldsymbol{u} t(n, m)$ at the current moment time $k+1$ and the state $\varphi(n, m, k)$ the same cell A $\boldsymbol{u} t(n, m)$ and the states of the four neighboring cells $\varphi(n+1, m, k), \quad \varphi(n-1, m, k)$, $\varphi(n, m+1, k), \varphi(n, m-1, k)$ at the previous moment time $k$.

\section{Results and Discussion}

\subsection{The Schrodinger-Euclidean metamedium}

We can write the Schrödinger equation (2) in the idempotent basis $\left\{\mathbf{e}_{l u m}, \mathbf{E}_{c h r}\right\}$. Because

$$
\begin{aligned}
& \varphi(x, y, t)=\varphi_{r}(x, y, t)+\varphi_{g}(x, y, t) \varepsilon^{1}+\varphi_{b}(x, y, t) \varepsilon^{2}=\varphi_{l u m}(x, y, t) \cdot \mathbf{e}_{l u m}+\varphi_{c h r}(x, y, t) \cdot \mathbf{E}_{c h r}, \\
& f(x, y, t)=f_{r}(x, y, t)+f_{g}(x, y, t) \varepsilon^{1}+f_{b}(x, y, t) \varepsilon^{2}=f_{l u m}(x, y, t) \cdot \mathbf{e}_{l u m}+f_{c h r}(x, y, t) \cdot \mathbf{E}_{c h r}, \\
& \mathrm{D}=r+g \varepsilon^{1}+b \varepsilon^{2}=D_{l u m} \cdot \mathbf{e}_{l u m}+D_{c h r} \cdot \mathbf{E}_{c h r},
\end{aligned}
$$

the equation (2) breaks down onto two equations:
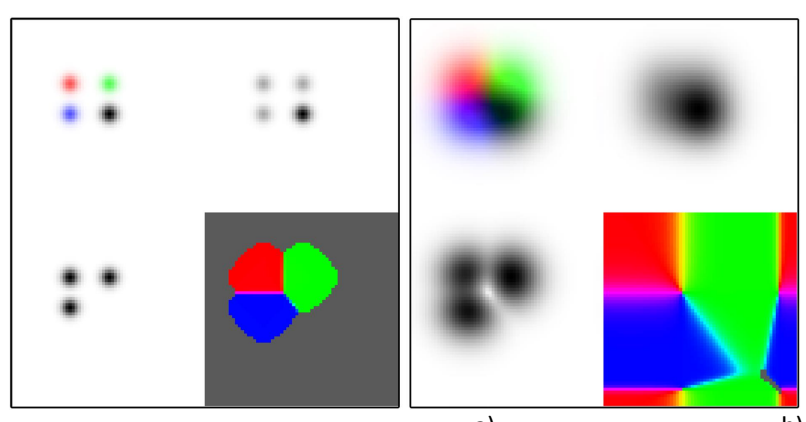

a)

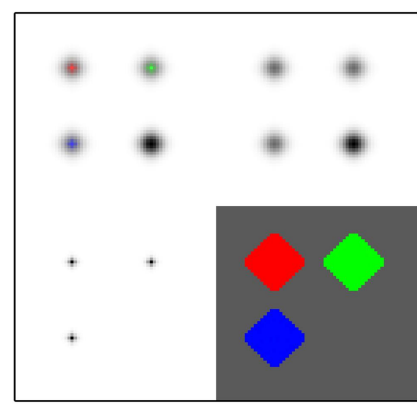

c)

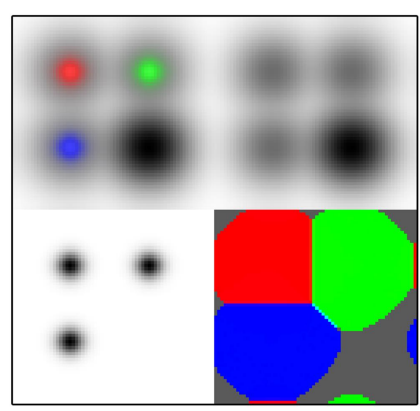

d)

Fig. 3. The state of the color Schrodinger-Euclidean metamedium at moments a) $t_{k}=16$ and b) $t_{k}=210$, when $D_{l u m}=S_{c h r}$, $\theta_{c h r}=0$ and c) $t_{k}=13$ and d) $t_{k}=120$, when $D_{l u m} \leq S_{c h r}, \theta_{c h r}=0$. 


$$
\begin{aligned}
& \frac{d}{d t} \varphi_{l u m}(x, y, t)=D_{l u m} \cdot\left(\frac{d^{2}}{d x^{2}} \varphi_{l u m}(x, y, t)+\frac{d^{2}}{d y^{2}} \varphi_{l u m}(x, y, t)\right), \\
& \frac{d}{d t} \varphi_{c h r}(x, y, t)=D_{c h r} \cdot\left(\frac{d^{2}}{d x^{2}} \varphi_{c h r}(x, y, t)+\frac{d^{2}}{d y^{2}} \varphi_{c h r}(x, y, t)\right) .
\end{aligned}
$$

one for a luminance and other - for a chromatic components.

Obviously, $\varphi_{c h r}(x, y, t)=\left|\varphi_{c h r}(x, y, t)\right| e^{i \cdot \theta_{d r}(x, y, t)}=S(x, y, t) e^{i \cdot H_{d r}(x, y, t)}$, where $S(x, y, t)=\left|\varphi_{c h r}(x, y, t)\right|, H_{c h r}(x, y, t)=\theta_{c h r}(x, y, t)$ are the saturation and the hue of a wave function, respectively. The relation between an angle $\theta_{c h r}(x, y, t)$ and a color hue $H_{c h r}(x, y, t)$ is shown on a Fig. 2. The first expression in (7) is the equation of a heat conduction with a real-valued diffusion coefficient $D_{l u m}=r_{D}+g_{D}+b_{D}$. It describes the time brightness evolution $\varphi_{l u m}(x, y, t)$ of a wave function $\varphi(x, y, t)$. The second expression appears to be the Schrodinger equation with a complex diffusion coefficient $D_{c h r}=\left(r_{D}-\frac{g_{D}+b_{D}}{2}\right)+i_{-} \frac{\sqrt{3}}{2}\left(g_{D}-b_{D}\right)$. It describes the time hue evolution $\varphi_{c h r}(x, y, t)$ of the wave function.

For the modeling results representation we will use the cellular automaton, in which the cell's states are shown as color pixels (as triplet numbers). The sum of four Dirac's delta-functions (red, green, white and blue) as an input signal $f(x, y, t)$. On Fig. 3 these functions are represented as four points of the corresponding colors. Each figure consist of four parts: the top left quarter shows the resulting RGB picture (i.e. presents wave function $\varphi(x, y, t)$ in the RGB format), the top right part shows the luminance component $\varphi_{\text {lum }}(x, y, t)$ of a color wave function, the bottom left one shows the saturation $\left|\varphi_{c h r}(x, y, t)\right|=S(x, y, t)$ and the last one represents the color tone $\theta_{c h r}(x, y, t)$.

Initially we will consider time evolution of the Schrodinger-Euclidean metamedium for the "balanced" chromatic and achromatic parameters $D=D_{l u m} \cdot \mathbf{e}_{l u m}+S_{c h r} \cdot e^{i-H_{c h r}} \cdot \mathbf{E}_{c h r}$ where $D_{l u m}=S_{c h r}, \theta_{c h r}=0$, i.e. $D=D_{l u m} \cdot\left(\mathbf{e}_{l u m}+\mathbf{E}_{c h r}\right)$. In this case we take the equal values of a diffusion coefficient's luminance and saturation, when the chromatic phase is equal to zero: $D_{\text {lum }}=S_{c h r}, \theta_{c h r}=0$. The results of a simulation for this case are shown on Fig. 3a $\left(t_{k}=16\right)$ and Fig. 3b $\left(t_{k}=120\right)$. Fig. 3c-d shows the process of a color excitement's propagation in a color metamedium, which diffusion coefficient has the low value of saturation $\left(D_{l u m} \leq S_{c h r}, \theta_{c h r}=0\right)$. The achromatic components on all illustrations in this work are inverted to reduce the amount of dark colors for a better visual perception of pictures. Therefore, the darker colors mean higher values of excitement. Note that chromatic parts of all spots are spreading slower than achromatic ones: the size of spots in the top right quad (excitement's luminance representation) is bigger than in the bottom left one (excitement's saturation representation).

Results that are more interesting can be obtained when we increase the value of a color hue $\theta_{c h r}$ of a diffusion coefficient. As an input signal we use a single red-colored Dirac's delta-function that is affecting the central point of a cellular automaton. For a comparison, it is important to see the excitement of cellular automaton with a zero color hue $\theta_{c h r}=0^{\circ}$ (when $D_{l u m}=S_{c h r}=0.11$ ). The results are presented on Fig. 4. This picture shows only resulting RGB images (the top part) and cells' chromatic phases (the bottom part). Also, note the Fig 5.
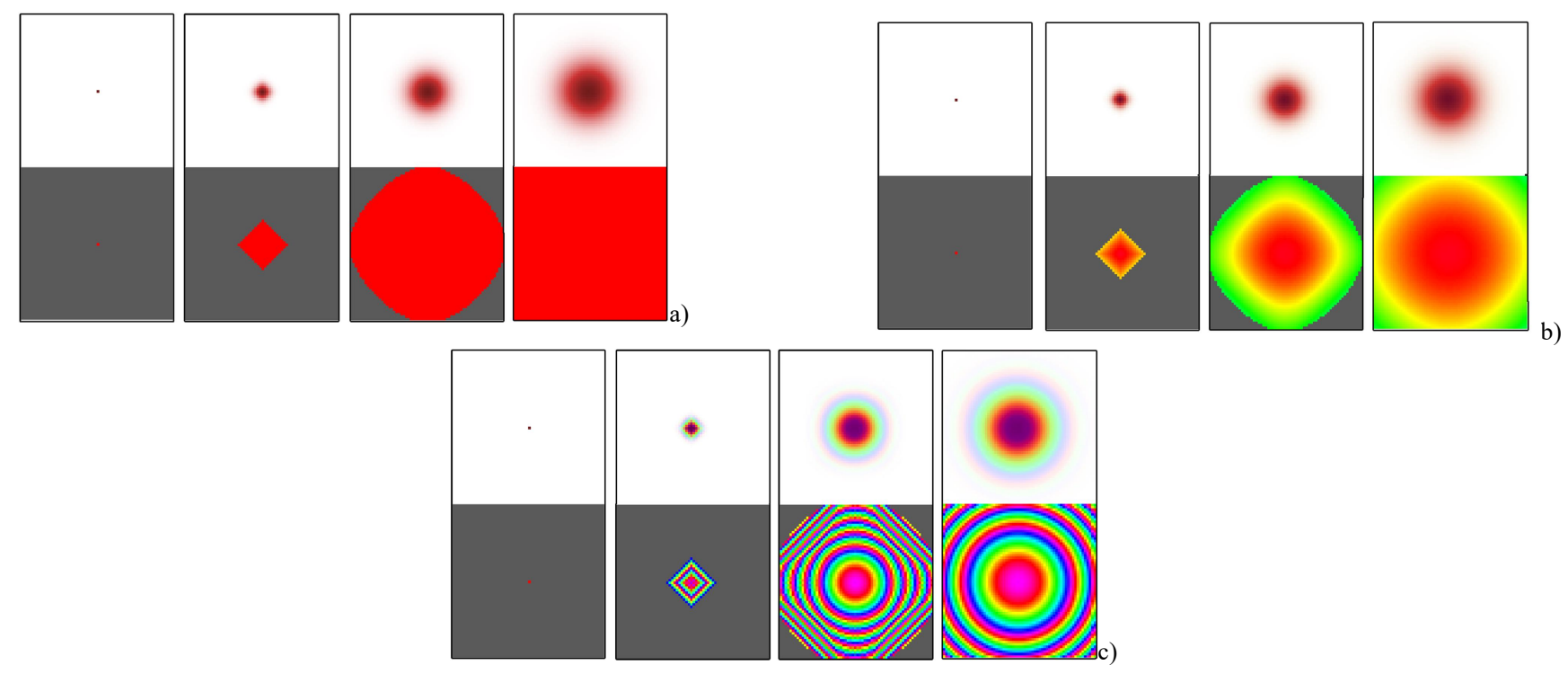

Fig. 4. The state of time evolution of the color Schrodinger-Euclidean metamedium at moments $t_{k}=0,10,70,160$

$$
\text { a) } \left.\left.\theta_{c h r}=0^{\circ}, b\right) \theta_{c h r}=5^{\circ}, c\right) \theta_{c h r}=60^{\circ} \text { ). }
$$




\subsection{The Schrodinger-Yaglom color metamedia}

The chromatic plane, in which $D_{c h r}=\left|D_{c h r}\right| e^{i_{-} \cdot \theta_{c h r}}=S_{c h r} \cdot e^{i_{-} \cdot H_{c h r}}$ lays, appears to be a classic complex algebra with $i_{-}^{2}=-1$. It is interesting to study a color metamedium with a chromatic plane in the form of another two complex algebra with $i_{+}^{2}=+1$ and $i_{0}^{2}=0[17]$, i.e. with the following chromatic components:

$$
D_{c h r}=\left|D_{c h r}\right| e^{i_{+} \cdot \theta_{c h}}=S_{c h} \cdot e^{i_{+} \cdot H_{c h}} \text { and } D_{c h r}=\left|D_{c h r}\right| e^{i_{0} \cdot \theta_{c h}}=S_{c h} \cdot e^{i_{0} \cdot H_{c h}} \text {. }
$$

We will call such media the color Schrodinger-Yaglom metamedia. The Fig. 6a contains excitements for color SchrodingerGalilean metamedium at the moment of time $t_{k}=128$ (for the same input signal as in the previous case) for different values of the hue of the diffusion coefficient $\left(\theta_{c h r}=5^{\circ}, 20^{\circ}, 40^{\circ}, 60^{\circ}\right)$. As we can see on a Fig. $6 \mathrm{~b}$, the further increase of the hue (for diffusion coefficient) $\theta_{c h r}=70^{\circ}, 80^{\circ}, 89^{\circ}, 90^{\circ}$ leads to the fast concentration and contraction of a phase circle in the middle of the bottom right square. In addition, our red-colored initial point completely turns into a spot with a pearl halo when the hue of the coefficient $D$ reaches $\theta_{c h r}=90^{\circ}$. In addition, we should mention that values $\theta_{c h r}=\arg \left\{D_{c h r}\right\}$ do not produce any new phenomena because of the periodic nature of trigonometric functions. Indeed, the color excitement with $\arg \left\{D_{c h r}\right\}=\theta_{c h r}>90^{\circ}$ turns out to be the inverted (by a color tone) excitement of a metamedium with $\arg \left\{D_{c h r}\right\}=\theta_{c h r}-90^{\circ}$ (see the Fig. 7a that is quite similar to Fig. 6b).

The example of the excitement of the Schrödinger-Minkowskian metamedium with a chromatic component of a diffusion coefficient in the form of a double number $D_{c h r}=\left|D_{c h r}\right| e^{i_{+} \cdot \theta_{c r}}=S_{c h r} \cdot e^{i_{+} \cdot H_{c h}}$ is shown on a Fig. 7b.
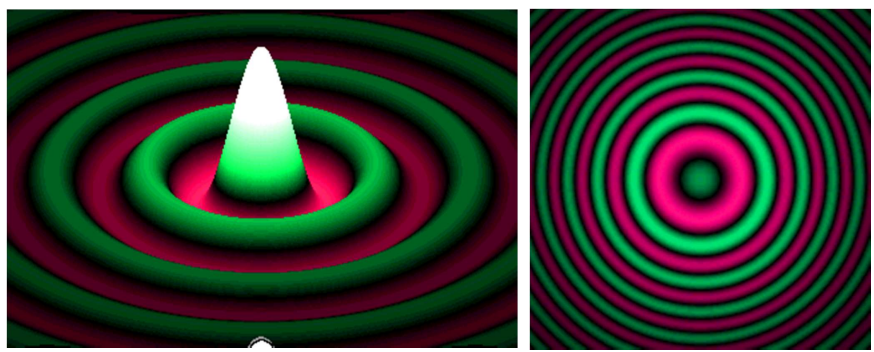

Fig. 5. The typical form of an excitement for Schrodinger-Euclidean metamedium under the impact of an input white Dirac's delta-impulse.

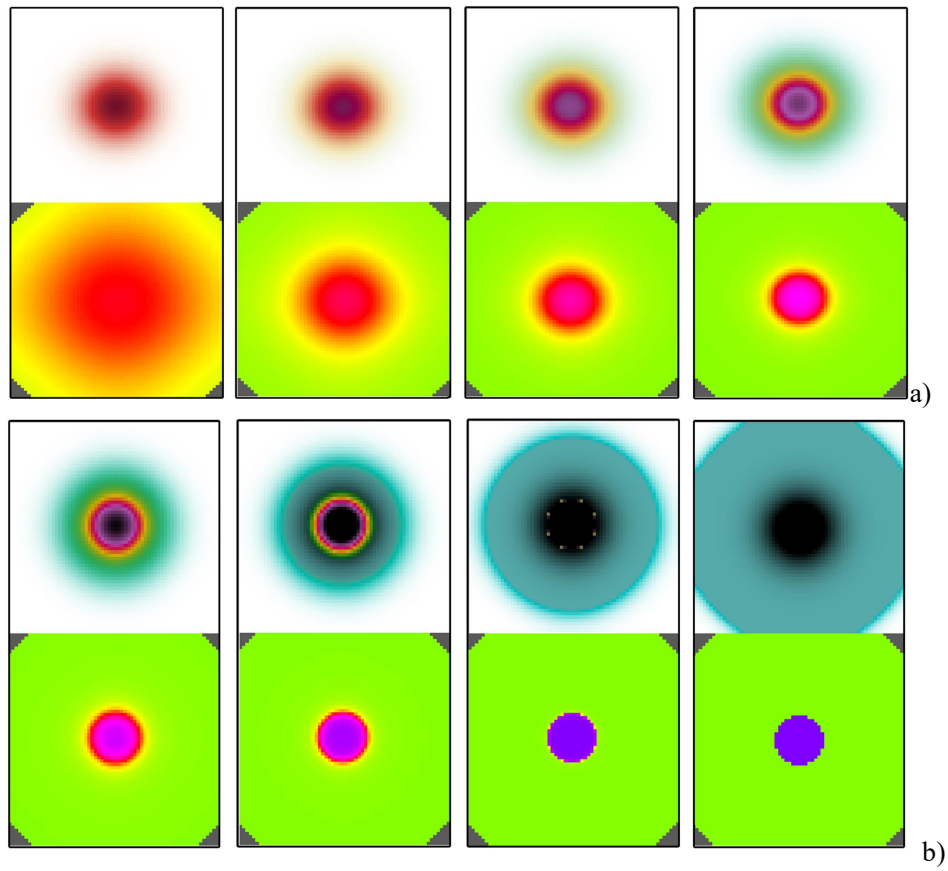

Fig. 6. The state of time evolution of the color Schrodinger-Galilean metamedium $\left(i^{2}=0\right)$ at the moment $t_{k}=128:$ a) $\theta_{c h r}=5^{\circ}, 20^{\circ}, 40^{\circ}, 60^{\circ}$, b) $\theta_{c h r}=70^{\circ}, 80^{\circ}, 89^{\circ}, 90^{\circ}$.

\subsection{The excitement of the color Schrödinger metamedium by a moving source}

Let the excitement function $f(x, y, t)$ in equation (2) be the Dirac delta-function that is moving on the circle with a radius $R$ and the center at the point $\left(x_{0}, y_{0}\right)$. The source has an angular velocity $\Omega$ : 
$f(x, y, t)=\delta\left(x_{0}+R \cdot \cos (\Omega \cdot t), y_{0}+R \cdot \sin (\Omega \cdot t)\right)$, where $\left(x_{0}-x(t)\right)^{2}+\left(y_{0}-y(t)\right)^{2}=R^{2}$. It means that we have a moving quantum particle in a color metamedium. Firstly, we will research the color Schrodinger-Euclidean metamedium with the chromatic component in the form of a classical complex number $D_{c h r}=\left|D_{c h r}\right| e^{i_{-} \cdot \theta_{c h r}}=S_{c h r} \cdot e^{i_{-} \cdot H_{c h r}}$ that has a relatively low chromatic phase value $\theta_{c h r}$ of $D_{c h r}$. The Fig.8a-b demonstrates the results of modeling for this case.
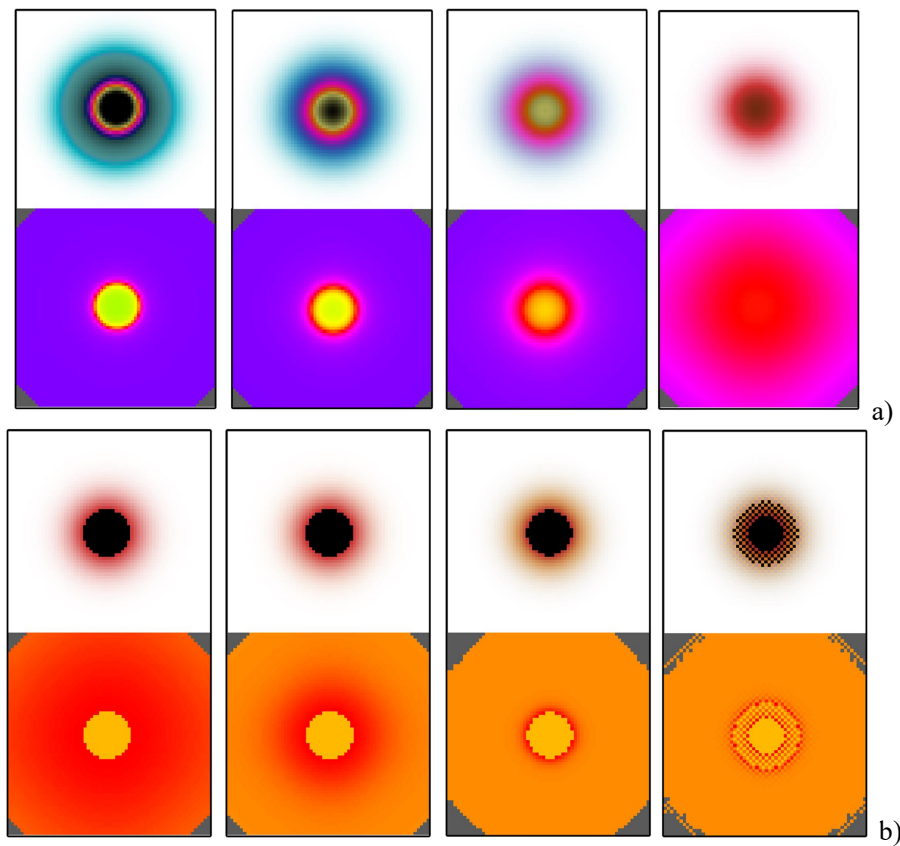

Fig. 7. a) The excitement of a color Schrödinger-Galilean metamedium $\left(i^{2}=0\right)$ at the moment $t_{k}=128$ for the diffusion coefficients with hues $\theta_{c h r}=100^{\circ}, 110^{\circ}, 130^{\circ}, 175^{\circ}$; b) The excitement of the color Schrödinger-Minkowskian metamedium $\left(i^{2}=+1\right)$ at the moment $t_{k}=128$ for the diffusion coefficients with hues $\theta_{c h r}=100^{\circ}, 110^{\circ}, 130^{\circ}, 175^{\circ}$.

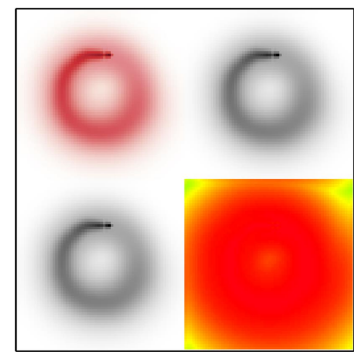

a) $\theta_{c h r}=5^{\circ}$

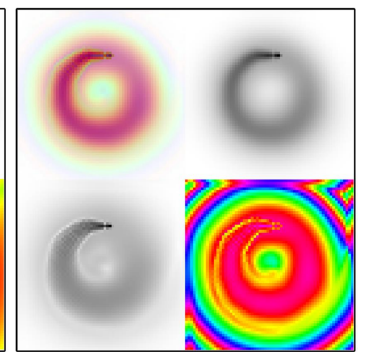

b) $\theta_{c h r}=60^{\circ}$

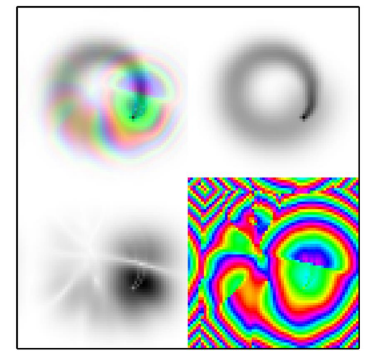

c) $\theta_{c h r}=74^{\circ}$

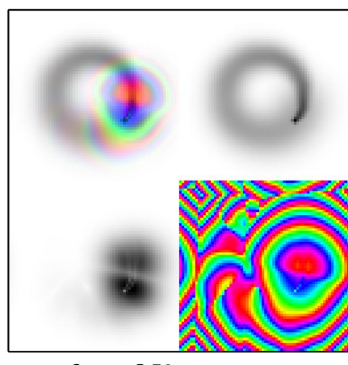

d) $\theta_{c h r}=85^{\circ}$

Fig. 8. The excitement of the color Schrödinger-Euclidean metamedium by a particle moving on a circular trajectory $\left(t_{k}=128\right)$.

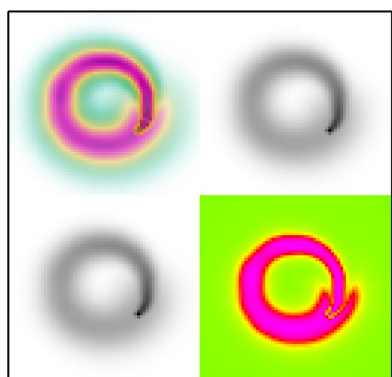

a) $\theta_{c h r}=70^{\circ}$

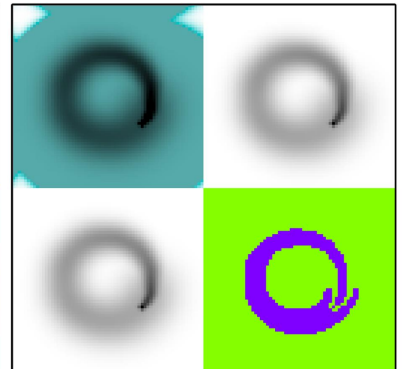

b) $\theta_{c h r}=90^{\circ}$

Fig. 9. The excitement of the Schrödinger-Galilean metamedium by a particle moving on a circle $\left(t_{k}=100, \quad i_{0}^{2}=0\right)$.

When the chromatic angle $\theta_{c h r}$ values are small (low color tone) then $D_{c h r}$ 's excitement fluctuation components, that are perpendicular to the movement trajectory, are almost absent. We only can see the parts of fluctuations that exist along the trajectory. When values of the chromatic angle $\theta_{c h r}$ (hue) are being increased, we can observe the excitement's fluctuations that are perpendicular to the trajectory of a movement. Also the interference of a "tail" and "head" parts becomes visible (see Fig. 8cd). 
Image Processing, Geoinformation Technology and Information Security / V. Labunets et al.

Different results can be obtained for color media with a chromatic component in the form of a double $D_{c h r}=S_{c h r} \cdot e^{i_{0} \cdot \theta_{c h r}}$ and a dual $D_{c h r}=S_{c h r} \cdot e^{i_{0} \cdot \theta_{c h r}}$ number. For example, when we use a dual number $D_{c h r}=S_{c h r} \cdot e^{i_{0} \cdot \theta_{c h r}}$ the alteration of $\theta_{c h r}=\arg \left\{D_{c h r}\right\}$ leads to some interesting and even more unusual consequences. The Fig. 9 shows the pictures of an excitement at the moment $t_{k}=100$ for the quite high values of a phase $\theta_{c h r}$ (the picture of an excitement changes weakly for the wide range of $\theta_{c h r}$ 's values). It can be seen that in the case of a Schrödinger-Galilean metamedium, the growth of a $D_{c h r}$ 's phase causes the increase of a violet and pearl color amounts (on condition that the moving particle has a red color). Particle trail's halo on the right part of Fig. 9 is quite bright, but there are no cells with high lightness and saturation parameter values in the investigated area. It is caused by irregular laws of the behavior of the chromatic component for this metamedia type.
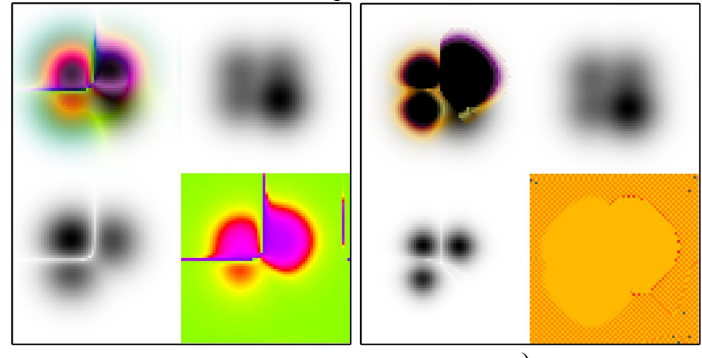

a)

b)

Fig. 10. The interference of four excitements at the moment $t_{k}=128$ in a) the Schrodinger-Galilean metamedium $\left(i^{2}=0\right)$ and b) the Schrödinger-

Minkowskian metamedium $\left(i^{2}=1\right)$ metamedia $\left(\theta_{c h r}=50^{\circ}\right.$ in both cases).

\subsection{The interference of excitements in the color Schrödinger metamedia}

The process of excitement's interference in Schrödinger-Euclidean metamedia has a classic character. The results of a simulation for Schrodinger-Galilean $\left(i^{2}=0\right)$ and Schrödinger-Minkowskian $\left(i^{2}=1\right)$ metamedia are shown on Fig. 10a and Fig. 10b, respectively. It can be seen on Fig. 10a that in the Schrödinger-Galilean metamedium the collision of different-colored excitements produces unusual rays in the areas where an occlusion happened. There are no such phenomena in the SchrodingerEuclidean and in the Schrödinger-Minkowskian metamedia.

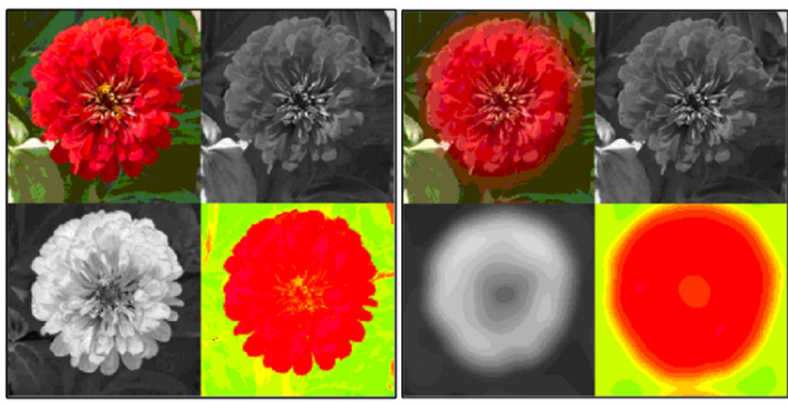

a)

b)

Fig. 11. a) The excitement function (input image) $f\left(x, y, t_{0}=0\right)$ of the color Schrödinger-Euclid metamedium at the initial moment $t_{0}=0$; b) The excitement of this metamedium at the moment $t_{k}=128$. The metamedium has broken the image onto the areas of uniformity with respect to brightness and hue at this time.

\subsection{Some applications of the color Schrödinger metamedia}

Let the excitement function $f\left(x, y, t_{0}=0\right)=f_{r}(x, y, 0)+f_{g}(x, y, 0) \varepsilon+f_{b}(x, y, 0) \varepsilon^{2}=f_{l u m}(x, y, 0) \cdot \mathbf{e}_{l u m}+f_{c h r}(x, y, 0) \cdot \mathbf{E}_{c h r}$ in (2) represents a color RGB image at the moment $t_{0}=0$. Then the color wave function

$$
\varphi(x, y, t)=\varphi_{r}(x, y, t)+\varphi_{g}(x, y, t) \varepsilon+\varphi_{b}(x, y, t) \varepsilon^{2}=\varphi_{l u m}(x, y, t) \cdot \mathbf{e}_{l u m}+\varphi_{c h r}(x, y, t) \cdot \mathbf{E}_{c h r}
$$

shows us the time evolution of initial image $f\left(x, y, t_{0}=0\right)=\varphi(x, y, 0)$. As an example of such image, we take a flower in an RGB format (see Fig. 11b, top left quarter). The luminance component $\varphi_{\text {lum }}(x, y, t)$ of wave function (8) represented in the bottom left part of Fig. 11b, the saturation component $\left|\varphi_{c h r}(x, y, t)\right|-$ in the top right quarter and the hue $\theta(x, y, t)=\arg \left\{\varphi_{c h r}(x, y, t)\right\}$ - in the bottom right part.

One of the most important tasks in the digital processing of color images [18] is the distinguishing of image's parts, where some of its components have uniform values. It is the uniformity areas detection, for example, we can detect the areas with a similar brightness, saturation or color tone, etc. Usually one has to perform such operation before starting the image segmentation by some parameter. It turns out that color Schrödinger metamedia are able to implement such operations. Fig. $11 \mathrm{~b}$ 
Image Processing, Geoinformation Technology and Information Security / V. Labunets et al.

shows the excitement of a Schrödinger-Euclidean metamedia at the moment $t=128$ after an impact that is represented as an image, which was described previously. It is easy to see that by this time the metamedia has broken the initial image onto areas of uniformity by luminance and by color tone. Fig. 12 and Fig. 13 shows the excitements of the Schrödinger-Galilean and Schrödinger-Minkowskian metamedia at the moments $t_{k}=0,32,64,128,160$ and $t_{k}=0,84$, respectively, after an input impact in the form of an initial image.

\section{Conclusion}

The metamedia with triplet (color) diffusion coefficients were first studied. Their laws of functioning are described by color Schrodinger equations. Simulation of these equations in the form of quantum cellular automata was considered. The results of modeling that were shown in this work demonstrate the complex character of the time evolution of such metamedia. Our future work will be focused on using commutative and Clifford algebras for hyperspectral image processing and pattern recognition.
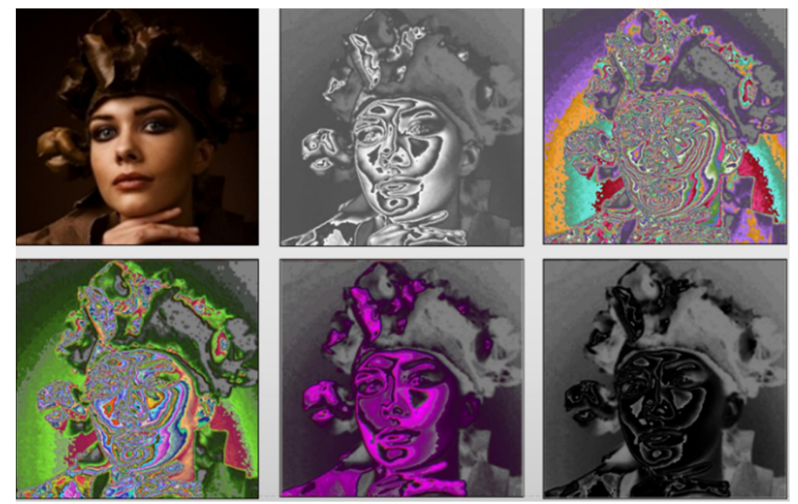

Fig. 12. The excitement of a Schrödinger-Galilean metamedia at moments of time $t_{k}=0,32,64,128,160$ when an input signal had the form of the initial image in the top left quarter.
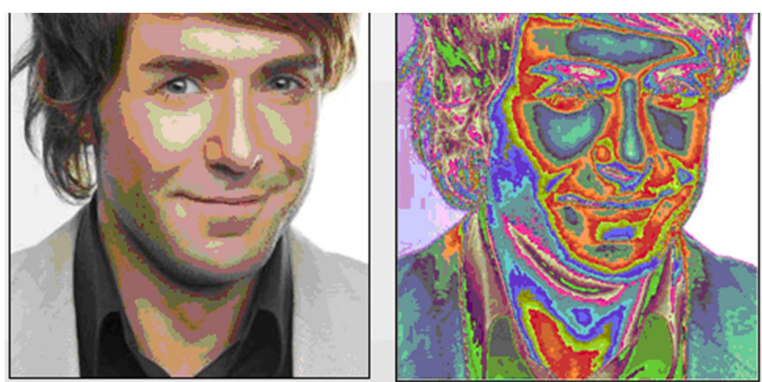

a)

b)

Fig. 13. The excitement of a Schrödinger-Minkowskian at the moment of time $t_{k}=0,84$ when an input signal had the form of an image.

\section{Acknowledgements}

This work was supported by grants the RFBR № 17-07-00886, № 17-29-03369 and by Ural State Forest University Engineering's Center of Excellence in "Quantum and Classical Information Technologies for Remote Sensing Systems".

\section{References}

[1] Nagasawa M. Schrodinger equations and diffusion theory. Monographs in mathematics. Birkheauser Verlag, Basel, Switzerland1993; 86: 238 p.

[2] Lou L, Zhan X, Fu Z, Ding M. Method of Boundary Extraction Based on Schrödinger Equation. Proceedings of the 21th Congress of the International Society for Photogrammetry and Remote Sensing - ISPRS. Beijing, China 2008; B5(2): 813-816.

[3] Hagan S, Hameroff SR, Tuzyinski JA. Quantum Computation in Brain Microtubules. Decoherence and Biological Feasibility, Physical Review E, American Physical Society 2002; 65: 1-11.

[4] Perus M, Bischof H, Caulfield J, Loo CK. Quantum Implementable Selective Reconstruction of High Resolution Images. Applied Optics 2004; 43: 61346138.

[5] Rigatos GG. Quantum Wave-Packets in Fuzzy Automata and Neural Associative Memories. International Journal of Modern Physics C, World Scientific 2007; 18(9): 209-221.

[6] Greaves Ch. On algebraic triplets. Proc. Irisn Acad. 1847; 3: 51-54, 57-64, 80-84, 105-108.

[7] Wolfram S. Cellular automata as models of complexity. Reprinted from Nature. Macmillan Journals Ltd 1985; 311(5985): 419-424.

[8] Labunets V. Excitable Schrodinger metamedia. 23rd Internation Crimean Conference. Microwave and Telecommunication Technology. Conference proceedings 2013; I: 12-16.

[9] Obeid I, Morizi J, Moxon K, Nicolelis MA, Wolf PD. Two Multichannel Integrated Circuits for Neural Recording and Signal Processing. IEEE Trans Biomed. Eng. 2003; 50: 255-258. 
Image Processing, Geoinformation Technology and Information Security / V. Labunets et al.

[10] Harrison R, Watkins P, Kier R, Lovejoy R, Black D, Normann R, Solzbacher F. A Low-Power Integrated Circuit for a Wireless 100-Electrode Neural Recording System. International Solid State Circuits Conference 2006; Session 30.

[11] Ruedi PF, Heim P, Kaess F, Grenet E, Heitger F, Burgi P-Y, Gyger S, Nussbaum P. A 128 128, pixel 120-dB dynamic-range vision-sensor chip for image contrast and orientation extraction. IEEE J. Solid-State Circuits 2003; 38: 2325-2333.

[12] Lichtsteiner P, Posch C, Delbruck T. A 128128120 dB 30mW asynchronous vision sensor that responds to relative intensity change. IEEE J. Solid-State Circuits 2008; 43: 566-576.

[13] Zaghloul K, Boahen K. A. Optic nerve signals in a neuromorphic chip: Part 1. IEEE Trans. Biomed Eng. 2004; 51: 657-666.

[14] Zaghloul K, Boahen K. A. Optic nerve signals in a neuromorphic chip: Part 2. IEEE Trans. Biomed Eng. 2004; 51: 667-675.

[15] Mojarradi M, Binkley D, Blalock B, Andersen R, Uslhoefer N, Johnson T, Del Castillo L. A miniaturized neuroprosthesis suitable for implantation into the brain. IEEE Trans. Neural Syst. Rehabil. Eng. 2003; 11: 38-42.

[16] Labunets V, Artemov I, Chasovskikh V, Ostheimer E. Retinamorphic bichromatic Schrödinger metamedia. CEUR Workshop Proceedings 2017; 1901: 140148. DOI: 10.18287/1613-0073-2017-1901-140-148.

[17] Yaglom I. Complex numbers in geometry. New York: Academic Press 1968; 242: 203-205.

[18] Rosin P, Adamatzky A, Sun X. Cellular Automata in Image Processing and Geometry. Switzerland: Springer International Publishing 2014 ; 65-80. 